\title{
A LIMITING ABSORPTION PRINCIPLE FOR SEPARATED DIRAC OPERATORS WITH WIGNER VON NEUMANN TYPE POTENTIALS
}

\author{
HORST BEHNCKE* AND PETER REJTO ${ }^{\dagger}$
}

1. Introduction. Several authors formulated a limiting absorption principle for several classes of operators. For the physical significance of this principle we refer to the paper of Eidus [Ei] and for brevity, for additional references we only refer to the AMS Memoir of Ben-Artzi and Devinatz [BD].

In this paper we establish the principle of limiting absorption for a class of separated Dirac operators corresponding to a class of potentials which contains the one of von Neumann - Wigner [VW], [RS].

In Section 2 we define this class of potentials and formulate our conditions on the intervals. Then in Theorem 2.1, which is our main theorem, we state the principle of limiting absorption. We prove Theorem 2.1 in the four sections that follow.

In Section 3 we construct approximate solutions to our basic system near infinity. The result of this construction is described in Theorem 3.1. To prove Theorem 3.1, first we follow Levinson [Ea] and in Lemma 3.2 we diagonalize the long range part of the potential. Then in Lemma 3.3 we follow Harris-Lutz [HL] and with the help of an approximate solution to a Riccati equation we achieve that the 21-element of the resulting system is short range. To prove Lemma 3.3 we adapt the notion of a slowly varying function to our class of potentials. Then we seek an approximate solution to the basic Riccati equation with the help of slowly varying functions. This construction is similar to the ones of [R], [DR] and [DMR].

In Section 4 we formulate estimates for the solutions of the basic system near zero. First, we construct approximate solutions and then use a result of Love-Erdelyi-Olver $[\mathrm{L}],[\mathrm{Er}],[\mathrm{O}],[\mathrm{RT}]$ to show that the same estimates hold for the exact solutions.

In Section 5 we formulate estimates for the basic system near infinity. In Theorem 5.1 we show that the exact solutions satisfy the same estimates as the approximate solutions of Theorem 3.1.

In Section 6, in Theorem 6.1 we formulate the weighted resolvent estimates which imply the principle of limiting absorption. The proof of Theorem 6.1 is based on the Weyl-Weidmann formula [We] for the resolvent kernel and on the Schur-Holmgren-Carleman bound of an integral operator $[\mathrm{F}],[\mathrm{Ok}]$. Since this construction is simpler under the additional assumption (6.3), in this section we assume it. In Theorem 6.2 we choose a weight and then show that the Schur-Holmgren-Carleman bound of the weighted

* Fachbereich Mathematik, 4500 Osnabrück, Germany.

$\dagger$ School of Mathematics, University of Minnesota, Minneapolis, MN 55455. 
resolvent operator with respect to this weight, is uniformly bounded. Here we use the same Schur-Holmgren-Carleman weight as in [LR]. To prove Theorem 6.2, we need global upper estimates for each of these two normalized solutions and a lower estimate for their Wronskian. These estimates are formulated in Lemma 6.3. We prove the upper estimates by giving upper estimates for the connection constants. We prove the lower estimates using an adaptation of Lemma 3.3 of [DMR].

In Section 7 we complete the proof of Theorem 6.1 and hence of the main Theorem 2.1 by removing the additional assumption (6.3).

For recent work on absolute continuity we refer to the papers of Behncke $[\mathrm{B}],[\mathrm{B}]$, Gilbert-Pearson [GP] and [St]. For the theory of embedded halfbound states we refer Hinton-Klaus-Shaw [HKS] and for the question of asymptotics near resonance points we refer to the paper of Klaus [K].

It is a pleasure to thank Professors Devinatz, Harris, McCarthy and the referee for their valuable suggestions.

2. Formulation of the result. We consider Dirac operators with spherically symmetric potentials and assume that they are real valued and locally integrable on $(0, \infty)$. The separated Dirac operator with electric potential $p$ and mass $m$ can be written, at least formally, as

$$
K=\left[\begin{array}{cc}
p-m & \ell / t \\
\ell / t & p+m
\end{array}\right]+D J
$$

where, $\ell= \pm 1 \pm 2, \ldots, J=\left[\begin{array}{cc}0 & -1 \\ 1 & 0\end{array}\right]$ and $D=\frac{d}{d t}$. Note that the off diagonal elements of this matrix are equal. Hence this operator $K$ is a particular case of an operator of the form,

$$
K=V+D J,
$$

where the matrix $V$ is of the form

$$
V=\left[\begin{array}{ll}
V_{1} & V_{2} \\
V_{2} & V_{3}
\end{array}\right]
$$

From such a formal operator $K$ selfadjoint operators can be derived in a standard fashion, see e.g. [We] and we call these selfadjoint operators Hamiltonians.

We start the formulation of the principle of limiting absorption for our Hamiltonians by formulating assumptions on the potentials. Specifically, we assume that in some neighborhood of infinity, say $(c, \infty)$, each $V_{j}$ admits a decomposition of the form,

$$
V_{j}=S_{j}+P_{j}+W_{j}, \quad j=1,2,3, \text { or } V=S+P+W,
$$

where the $S_{j}$ are short range in the sense that,

$$
S_{j} \in \mathcal{L}^{1}(c, \infty),
$$


and $P_{j}$ are long range in the sense that,

$$
P_{j}^{\prime} \in \mathcal{L}^{1}(c, \infty), \quad j=1,2,3 .
$$

Clearly, assumption (2.5) implies that the matrix potential $P$ converges to a limiting matrix $P(\infty)$ for $t \rightarrow \infty$, and we assume that $P(\infty)$ is such that,

$$
P_{1}(\infty)=-m, P_{3}(\infty)=m>0 \text { and } P_{2}(\infty)=0 .
$$

Then, we assume that each $W_{j}$ can be written in the form,

$$
W_{j}=f_{j} \sin g_{j}, \quad j=1,2,3 ;
$$

where, each $f_{j}$ is such that

$$
f_{j} \in \mathcal{L}^{2}(c, \infty) \text { and it is differentiable and } f_{j}^{\prime} \in \mathcal{L}^{1}(c, \infty)
$$

and $g_{j}$ is such that,

$$
\lim _{t \rightarrow \infty} g_{j}^{\prime}(t) \text { exists and } g_{j}^{\prime}(\infty)>0 \text {, or } g_{j}^{\prime}(t) \text { tends to infinity. }
$$

Furthermore, each $g_{j}$ is 2-times differentiable and

$$
\frac{g_{j}^{\prime \prime}}{\left(g_{j}^{\prime}\right)^{2}} f_{j} \in \mathcal{L}^{1}(c, \infty) .
$$

Note that these assumptions define a class of potentials which contains the oscillating part of the von Neumann Wigner potential,

$$
W_{j}(t)=(1+t)^{-1} \sin t
$$

Finally, we assume that on the interval $(0, c)$ the matrix $V$ admits a decomposition of the form

$$
V(t)=t^{-1} \cdot J C_{0}(e, \ell)+B(t)
$$

where

$$
C_{0}(e, \ell)=\left[\begin{array}{cc}
-e & \ell \\
\ell & -e
\end{array}\right], \quad e \in \mathbf{R}^{1},
$$

and

$$
\|B\|_{\mathcal{L}^{\infty}(0, e)}<\infty .
$$

We continue the formulation of the principle of limiting absorption for our Hamiltonians by formulating assumptions on the interval $\mathcal{I}$. First, we we assume that for each $\lambda \in \mathcal{I}$ the limit matrix, $P(\infty)-\lambda$ has two distinct imaginary eigenvalues;

$$
\operatorname{Spec}(P(\infty)-\lambda)=\{i \mu(\infty),-i \mu(\infty)\}, \mu(\infty) \neq 0, \mu(\infty) \in \mathcal{R}
$$


Our second assumption is that for each $j=1,2,3$,

$$
\inf _{t>c} \inf _{\lambda \in \mathcal{I}}\left|2 \mu(\infty)-g_{j}^{\prime}(t)\right|>0 .
$$

We complete the formulation of a principle of limiting absorption for our Hamiltonians by introducing the family of operators,

$$
F_{s}^{ \pm}(\lambda)=m_{s}^{1 / 2} R(\lambda) m_{s}^{1 / 2}, \operatorname{Im} \lambda \neq 0 .
$$

Here $R(\lambda)$ is the resolvent of a given selfadjoint extension of the formal operator of the definition (2.1),

$$
R(\lambda)=(\lambda I-K)^{-1}, \operatorname{Im} \lambda \neq 0,
$$

and $m_{s}$ is the operator of multiplication by the function,

$$
m_{s}(\xi)=\left(1+\xi^{2}\right)^{-s / 2}, \quad \xi \in \mathbf{R}^{+}, \quad s \in \mathbf{R} .
$$

THEOREM 2.1. Let the given potential $V$ satisfy assumptions (2.3) ... (2.14) and let the given interval $\mathcal{I}$ satisfy assumption (2.15) with respect to it. Then, for each selfadjoint extension of the operator of the definition (2.1) the principle of limiting absorption holds over the interval $\mathcal{I}$. More specifically, there are two continuous operator valued functions $F_{s}^{ \pm}(\lambda)$ of the variable $\lambda \in \mathcal{I}$ such that,

$$
\lim _{\epsilon=0} \sup _{\lambda \in \mathcal{R}_{ \pm}(\mathcal{I})}\left\|F_{s}^{ \pm}(\lambda)-F_{s}^{ \pm}(\lambda \pm \epsilon)\right\|=0 \quad s>1 / 2 .
$$

To motivate assumption (2.14) we observe that assumption (2.6) and formula (3.5), to be stated, show that for $\operatorname{Re} \lambda=\lambda$ assumption (2.14) is equivalent to

$$
|\operatorname{Re} \lambda|>m .
$$

This assumption, in turn, is equivalent to the assumption that for such a $\lambda$ the basic system corresponding to the operator (2.1),

$$
u^{\prime}=\left[\begin{array}{cc}
-V_{2} & -V_{3}+\lambda \\
V_{1}-\lambda & V_{2}
\end{array}\right] u=(V-\lambda J) u,
$$

has oscillatory solutions. In other words, $\lambda$ is in the continuous spectrum of the Hamiltonian.

We shall prove the main Theorem 2.1 in the four sections that follow. Our proof will make essential use of the Weyl-Weidmann formula [We], which for $\operatorname{Im} \lambda \neq 0$ allows us to study the resolvent of the definition (2.17) with the help of two solutions of the basic system (2.21) and their Wronskian. More specifically, this formula allows us to study the asymptotic properties of this resolvent kernel with the help of the asymptotic properties of two solutions of the basic system (2.21) and their Wronskian. The technical part of the study of the asymptotic properties of such solutions is isolated in the following Section 3 . In it, we construct approximate solutions to the basic system (2.21). 
3. Construction of approximate solutions to the basic system (2.21) near infinity.

THEOREM 3.1. Let the potential $V$ and interval $\mathcal{I}$ satisfy the assumptions of Theorem 2.1. Then for each $\lambda$ with Re $\lambda$ in $\mathcal{I}$ there are approximate solutions $z_{r}$ and $y_{r}$ to the system (2.21) in the sense that there is a matrix $E_{r}$ such that

$$
\sup _{\operatorname{Re} \in \mathcal{I}}\left\|E_{r}(., \lambda)\right\|_{\mathcal{L}^{1}(c, \infty)}=<\infty .
$$

and $z_{r}$ and $y_{r}$ satisfy,

$$
y_{r}^{\prime}=\left[\left(V-\lambda J+E_{r}\right)\right] y_{r} .
$$

Furthermore,

$$
y_{r} \in L^{2}\left((c, \infty), \mathcal{C}_{2}\right) \quad \text { for } \operatorname{Im} \lambda \neq 0 .
$$

We prove Theorem 3.1 by constructing an approximate fundamental matrix to the system $(2.21)$ by a repeated change of the dependent variable. As is well known if $u$ is a solution of the system (2.21) and if $A$ is a given smooth and invertible matrix, then the function $v=A^{-1} u$ satisfies,

$$
v^{\prime}=\left[A^{-1}(V-\lambda J) A-A^{-1} A^{\prime}\right] v .
$$

As a first step of the proof of Theorem 3.1 we follow Levinson [Ea] and choose $A$ to be a diagonalizing transformation for the the matrix $P-\lambda J$, the long range part of $V-\lambda J$. To do this, note that assumptions (2.6) and (2.14) allow us to choose a far out region $[c, \infty)$ so that for $t$ in $[c, \infty)$ this matrix has two distinct eigenvalues, say $\pm i \mu$. Then elementary algebra yields the formula,

$$
\mu=\mu(t, \lambda)=\left(\left(P_{1}(t)-\lambda\right)\left(P_{3}(t)-\lambda\right)-P_{2}^{2}(t)\right)^{1 / 2} .
$$

For future reference we choose the branch of the square root function so that,

$$
\text { Re } i \mu \leq 0 \text {, for } \operatorname{Im} \lambda \geq 0 \text {. }
$$

The lemma that follows formulates an elementary property of our class of potentials. It was also verified in [BR].

LEMMA 3.1. Let the potential $P$ and interval $\mathcal{I}$ satisfy the assumptions of Theorem 2.1. Then for each $\lambda$ with $\operatorname{Re} \lambda$ in $\mathcal{I}$ there is a transformation $A=A(t, \lambda)$ such that,

$$
A^{-1}(P-\lambda J) A=\operatorname{diag}(i \mu,-i \mu) .
$$

Furthermore,

$$
\sup _{\operatorname{Re\lambda \in \mathcal {I}}}\|A(., \lambda)\|_{\mathcal{L}^{\infty}(c, \infty)}+\sup _{\operatorname{Re} \lambda \in \mathcal{I}}\left\|A^{-1}(., \lambda)\right\|_{\mathcal{L}^{\infty}(c, \infty)}<\infty,
$$


and

$$
\sup _{\operatorname{Re} \lambda \in \mathcal{I}}\left\|A^{\prime}(., \lambda)\right\|_{\mathcal{L}^{1}(c, \infty)}<\infty
$$

Conclusions (3.9) and (3.8) of Lemma 3.1 allow us to choose $A$ in formula (3.4) by this lemma. For future reference we define:

$$
R(1)=A^{-1} S A-A^{-1} A^{\prime},
$$

and

$$
U(1)=\left[\operatorname{diag}(i \mu,-i \mu)+A^{-1} W A\right] .
$$

Then combining the definitions (3.11) and (3.10) with conclusion (3.7) and with assumption (2.3) we find,

$$
A^{-1}(V-\lambda J) A-A^{-1} A^{\prime}=U(1)+R(1) .
$$

Hence replacing $v$ by $u_{1}$ the system $(3.4)$ can be written in the form,

$$
u_{1}^{\prime}=[U(1)+R(1)] u_{1} .
$$

To motivate the definition (3.10) note that conclusions (3.8) and (3.9) together with assumption (2.4) show that $R(1)$ is short range. More specifically, they show that

$$
\sup _{\operatorname{Re\lambda } \in \mathcal{I}}\|R(1)(., \lambda)\|_{\mathcal{L}^{1}(c, \infty)}<\infty
$$

As a second step of the proof of Theorem 3.1 we choose $A$ in this formula so that the $(2,1)$ element of the system resulting from $(3.13)$ is short range. We do this with the help of a transformation suggested by Harris-Lutz [HL]. It is of the form $I+Q_{\ell}$ where

$$
Q_{\ell}=q_{\ell} \cdot\left[\begin{array}{ll}
0 & 0 \\
1 & 0
\end{array}\right]
$$

and the properties of $q_{\ell}$ are described in the lemma that follows.

LEMMA 3.2. Let the potentials $P, W$ and interval $\mathcal{I}$ satisfy the assumptions of Theorem 2.1 and let the matrix $U(1)$ be given by the definition (3.11). Then, for each $\lambda$ with $\operatorname{Re} \lambda \in \mathcal{I}$ there is a function $q_{\ell}=q_{\ell}(t, \lambda)$ such that, for the corresponding matrix function of the definition (3.15),

$(3.16) \sup _{\operatorname{Re} \lambda \in \mathcal{I}}\left\|\left[\left(I+Q_{\ell}\right)^{-1} U(1)\left(I+Q_{\ell}\right)-\left(I+Q_{\ell}\right)^{-1} Q_{\ell}^{\prime}\right]_{21}\right\|_{\mathcal{L}^{1}(c, \infty)}<\infty$.

Furthermore,

$$
\sup _{\operatorname{Re\lambda } \in \mathcal{I}}\left\|q_{\ell}(., \lambda)\right\|_{\mathcal{L}^{\infty}(c, \infty)}<\infty
$$


and

$$
\sup _{\operatorname{Re} \lambda \in \mathcal{I}}\left\|q_{\ell}(., \lambda)\right\|_{\mathcal{L}^{2}(c, \infty)}<\infty .
$$

We start the proof of conclusion (3.16) with the formula,

$$
\begin{aligned}
& {\left[\left(I+Q_{\ell}\right)^{-1} U(1)\left(I+Q_{\ell}\right)-\left(I+Q_{\ell}\right)^{-1} Q_{\ell}^{\prime}\right]_{21} } \\
= & -q_{\ell}^{\prime}+U(1)_{21}-2 U(1)_{11} q_{\ell}-U(1)_{12} q_{\ell}^{2} .
\end{aligned}
$$

To prove this formula, we show that the definition (3.15) implies that for each matrix $B$,

$$
\begin{gathered}
\left(I+Q_{\ell}\right)^{-1} B\left(I+Q_{\ell}\right)-\left(I+Q_{\ell}\right)^{-1} Q_{\ell}^{\prime}= \\
{\left[\begin{array}{cc}
B_{11}+q_{\ell} B_{12} & B_{12} \\
-q_{\ell}^{\prime}+B_{21}-q_{\ell}\left(B_{11}-B_{22}\right)-q_{\ell}^{2} B_{12} & B_{22}-q_{\ell} B_{12}
\end{array}\right]}
\end{gathered}
$$

To verify formula (3.20), note that the definition (3.15) yields,

$$
Q_{\ell}^{2}=0 \text { and so }\left(I+Q_{\ell}\right)^{-1}=I-Q_{\ell} .
$$

Formula (3.21), in turn, yields,

$$
\left(I+Q_{\ell}\right)^{-1} B\left(I+Q_{\ell}\right)=B+B Q_{\ell}-Q_{\ell} B-Q_{\ell} B Q_{\ell} .
$$

Similarly, it follows from the definition (3.15) that

$$
-\left(I+Q_{\ell}\right)^{-1} Q_{\ell}^{\prime}=-Q_{\ell}^{\prime} .
$$

Combining these two formula with the definition (3.15) we find formula (3.20). Then, applying formula (3.20) to the matrix $B=U(1)$ and using that according to the definition (3.11) trace $U(1)=0$, we find formula (3.19).

We continue the proof of conclusion (3.16) by constructing an approximate solution to the Riccati equation,

$$
-q_{\ell}^{\prime}+U(1)_{21}-2 U(1)_{11} q_{\ell}-U(1)_{12} q_{\ell}^{2}=0
$$

which we obtained by setting the right member of formula (3.19) equal to zero. Now we observe that there are functions $c_{ \pm j}^{r s}, r=1,2, s=1,2$, and $j=1,2,3$ with the property that for $r \neq s$

$$
U(1)_{r s}=\sum_{j=1}^{j=3}\left[c_{r s}^{+j} f_{j} \cdot \exp \left(i g_{j}\right)+c_{r s}^{-j} f_{j} \cdot \exp \left(-i g_{j}\right)\right],
$$

and each of these reduced coefficient functions is slowly varying in the sense that,

$$
\sup _{\operatorname{Re\lambda \in \mathcal {I}}}\left\|\left(c_{r s}^{ \pm j}\right)^{\prime}\right\|_{\mathcal{L}^{1}(c, \infty)}<\infty .
$$


To verify formula (3.23) we note that combining the definitions (3.11), (2.3) and (2.21) with assumption (2.7) we find constants, $\delta_{r s j k}$, such that,

$$
\begin{gathered}
U(1)_{r s}=\frac{1}{2 i} \cdot \sum_{j=1}^{j=3} \sum_{k=1}^{k=2} \delta_{r s j k} \cdot\left(A^{-1}\right)_{r j}\left[\exp \left(i g_{j}\right)-\exp \left(-i g_{j}\right)\right] A_{k s}, \\
\text { where } \delta_{r s j k}= \pm 1 \text { or } 0 .
\end{gathered}
$$

Hence formula (3.23) holds with

$$
c_{r s}^{ \pm j}= \pm \frac{1}{2 i} \cdot \sum_{k=1}^{k=2} \delta_{r s j k} \cdot\left(A^{-1}\right)_{r j} A_{k s} .
$$

To establish estimate (3.24), we combine this formula for the reduced coefficient functions with conclusions (3.9) and (3.8) of Lemma 3.2 and use that the product of two slowly varying functions is slowly varying. To see that the product of two slowly varying functions is slowly varying note that estimate (3.24) implies that for each $j$

$$
\sup _{\operatorname{Re} \lambda \in \mathcal{I}}\left\|c_{r s}^{ \pm j}(., \lambda)\right\|_{L^{\infty}(c, \infty)}<\infty .
$$

Similarly, it follows that

$$
U(1)_{11}=i \mu+\sum_{j=1}^{j=3}\left[c_{11}^{+j} f_{j} \cdot \exp \left(i g_{j}\right)+c_{11}^{-j} f_{j} \cdot \exp \left(-i g_{j}\right)\right]
$$

where, as before, the reduced coefficient functions $c_{11}^{ \pm j}$, are slowly varying. Inserting formula (3.23) into the Riccati equation (3.22) and using formula (3.25) to split the term $2 U(1)_{11} q_{\ell}$ into two we find,

$$
\begin{aligned}
-q_{\ell}^{\prime} & +\sum_{\substack{j=1 \\
j=3}}^{j=3}\left[c_{21}^{+j} f_{j} \cdot \exp \left(i g_{j}\right)+c_{21}^{-j} \cdot f_{j} \exp \left(-i g_{j}\right)\right]-2 i \mu q_{\ell} \\
-2 & \left.\sum_{\substack{j=1 \\
j=3}}^{+j} c_{11} \cdot \exp \left(i g_{j}\right)+c_{11}^{-j} f_{j} \cdot \exp \left(-i g_{j}\right)\right] q_{\ell} \\
& -\sum_{j=1}^{+j}\left[c_{12}^{+j} f_{j} \cdot \exp \left(i g_{j}\right)+c_{12}^{-j} f_{j} \cdot \exp \left(-i g_{j}\right)\right] q_{\ell}^{2}=0 .
\end{aligned}
$$

We seek an approximate solution to the Riccati equation (3.26) in the class of functions of the form,

$$
q_{\ell}=\sum_{j=1}^{j=3}\left[a^{+j} f_{j} \cdot \exp \left(i g_{j}\right)+a^{-j} f_{j} \cdot \exp \left(-i g_{j}\right)\right],
$$


where the reduced coefficient functions, $a^{ \pm j}$ are slowly varying;

$$
\sup _{\operatorname{Re} \lambda \in \mathcal{I}}\left\|\left(a^{ \pm j}\right)^{\prime}(., \lambda)\right\|_{\mathcal{L}^{1}(c, \infty)}<\infty
$$

Next we observe that assumption (2.8) implies that

$$
\begin{gathered}
f_{i} \cdot f_{j} \in \mathcal{L}^{1}(c, \infty) \text { and } f_{i} \cdot f_{j} \cdot f_{k} \in \mathcal{L}^{1}(c, \infty) \\
\quad \text { for }, i=1,2,3 ; j=1,2,3 ; k=1,2,3 .
\end{gathered}
$$

Combining estimates (3.29), (3.28) and (3.24) with assumption (3.27) we see that the second and third order terms in the functions $f_{j}$ are short range. Hence, inserting assumption (3.27) into the Riccati equation (3.26) and dropping these short range terms in the resulting equation we find,

$$
\begin{aligned}
& -\sum_{j=1}^{j=3}\left[a^{+j} i g_{j}^{\prime} f_{j} \cdot \exp \left(i g_{j}\right)-a^{-j} i g_{j}^{\prime} f_{j} \cdot \exp \left(-i g_{j}\right)\right] \\
& +\sum_{j=3}^{j=3}\left[c_{21}^{+j} f_{j} \cdot \exp \left(i g_{j}\right)+c_{21}^{-j} f_{j} \cdot \exp \left(-i g_{j}\right)\right] \\
& -2 \sum_{j=1}^{j=3}\left[i \mu a^{+j} f_{j} \cdot \exp \left(i g_{j}\right)+i \mu a^{-j} f_{j} \cdot \exp \left(-i g_{j}\right)\right]=0 .
\end{aligned}
$$

Rearranging the terms in equation (3.30) and setting the coefficients of each of the functions $\exp \left(i g_{j}\right)$ and $\exp \left(-i g_{j}\right)$ equal to zero leads to the formula, $\left(3.31_{ \pm}\right)$

$a^{ \pm j} f_{j}=\left(2 i \mu \pm i g_{j}^{\prime}\right)^{-1} f_{j} c_{21}^{ \pm j}$, or $a^{ \pm j}=\left(2 i \mu \pm i g_{j}^{\prime}\right)^{-1} \cdot c_{21}^{ \pm j}, \quad$ for $j=1,2,3$.

From now on we shall define these reduced coefficient functions by the second set of formula of $\left(3.31_{ \pm}\right)$.

We complete the proof of conclusion (3.16) by combining these assumptions with the ones of Theorem 2.1. More specifically, define

$$
e_{21}=\left[\left(I+Q_{\ell}\right)^{-1} U(1)\left(I+Q_{\ell}\right)-\left(I+Q_{\ell}\right)^{-1} Q_{\ell}^{\prime}\right]_{21}
$$

Then, since the left members of the equations (3.26) and (3.22) are equal, we see from the equation (3.30), from assumption (3.27) and from formula (3.19) that

$$
\begin{aligned}
e_{21}= & -\sum_{j=1}^{j=3}\left[\left(a^{+j} f_{j}\right)^{\prime} \cdot \exp \left(i g_{j}\right)+\left(a^{-j} f_{j}\right)^{\prime} \cdot \exp \left(-i g_{j}\right)\right] \\
& -2 \sum_{j=1}^{j=3}\left[c_{11}^{+j} f_{j} \cdot \exp \left(i g_{j}\right)+c_{11}^{-j} f_{j} \cdot \exp \left(-i g_{j}\right)\right] q_{\ell} \\
& -\sum_{j=1}^{j=3}\left[c_{12}^{+j} f_{j} \cdot \exp \left(i g_{j}\right)+c_{12}^{-j} f_{j} \cdot \exp \left(-i g_{j}\right)\right] q_{\ell}^{2}
\end{aligned}
$$


Estimates (3.29) and (3.24) together with assumption (2.8) show that the short range character of each of these three terms is implied by estimate (3.28). Combining assumptions (2.15), (2.10), (2.9), and (2.5) with formula $(3.5)$ we see that the first factors of the definitions $\left(3.31_{ \pm}\right)$are slowly varying. Since according to estimate $(3.24)$ so are the second factors, estimate (3.28) follows. Hence, each of the three terms in formula (3.33) is short range and this completes the proof of conclusion (3.16). Similarly, we see that assumptions (2.15), (2.9) and (2.8) imply conclusions (3.17) and (3.18). Thus, the proof of Lemma 3.2 is complete.

Applying formula (3.20) to the matrix $B=U(1)+R(1)$ and using the definition (3.32) we find,

$$
\begin{gathered}
\left(I+Q_{\ell}\right)^{-1}(U(1)+R(1))\left(I+Q_{\ell}\right)-\left(I+Q_{\ell}\right)^{-1} Q_{\ell}^{\prime}= \\
{\left[\begin{array}{cc}
(U(1)+R(1))_{11} & (U(1)+R(1))_{12} \\
e_{21}+R(1)_{21} & (U(1)+R(1))_{22}
\end{array}\right]+} \\
q_{\ell}\left[\begin{array}{cc}
(U(1)+R(1))_{12} & 0 \\
-R(1)_{11}+R(1)_{22}-q_{\ell} R(1)_{12} & -(U(1)+R(1))_{12}
\end{array}\right]
\end{gathered}
$$

Next define,

$R(2)=\left[\begin{array}{cc}R(1)_{11}+q_{\ell} R(1)_{12} & R(1)_{12} \\ e_{21}+R(1)_{21}-q_{\ell}\left(R(1)_{11}-R(1)_{22}\right)-q_{\ell}^{2} R(1)_{12} & R(1)_{22}-q_{\ell} R(1)_{12}\end{array}\right]$

and

$$
U(2)=\left[\begin{array}{cc}
U(1)_{11}+q_{\ell} U(1)_{12} & U(1)_{12} \\
0 & -\left(U(1)_{11}+q_{\ell} U(1)_{12}\right)
\end{array}\right]
$$

Then using that according to the definition (3.11) trace $U(1)=0$, we see from formula (3.34) and from the definitions (3.36) and (3.35) that

$$
\left(I+Q_{\ell}\right)^{-1}(U(1)+R(1))\left(I+Q_{\ell}\right)-\left(I+Q_{\ell}\right)^{-1} Q_{\ell}^{\prime}=U(2)+R(2) .
$$

We see from formula (3.37), in turn, that the transformation

$$
u_{2}=\left(I+Q_{\ell}\right)^{-1} u_{1}
$$

carries the system (3.13) into

$$
u_{2}^{\prime}=\left[U(2)+(R(2)] u_{2} .\right.
$$

As a third step of the proof of Theorem 3.1 we choose $A$ in this formula so that the (12) element of the system resulting from (3.39) is short range. We do this with the help of a transformation suggested by Harris-Lutz [HL]. It is of the form $I+Q_{u}$ where,

$$
Q_{u}=q_{u} \cdot\left[\begin{array}{ll}
0 & 1 \\
0 & 0
\end{array}\right]
$$


and $q_{u}$ will be an approximate solution of the linear differential equation (3.46) to be stated in the proof of the following lemma.

LEMMA 3.3. Let the potentials $P, W$ and interval $\mathcal{I}$ satisfy the assumptions of Theorem 2.1 and let the matrix $U(2)$ be given by the definition (3.36). Then, to each $\lambda$ with $R e \lambda \in \mathcal{I}$ there is a function $q_{u}=q_{u}(t, \lambda)$ such that, for the corresponding matrix function of the definition (3.40),

$$
\sup _{\operatorname{Re} \lambda \in \mathcal{I}}\left\|\left[\left(I+Q_{u}\right)^{-1} U(2)\left(I+Q_{u}\right)-\left(I+Q_{u}\right)^{-1} Q^{\prime} u\right]_{12}\right\|_{\mathcal{L}^{1}(c, \infty)}<\infty .
$$

Furthermore,

$$
\sup _{\operatorname{Re} \lambda \in \mathcal{I}}\left\|q_{u}(., \lambda)\right\|_{\mathcal{L}^{\infty}(c, \infty)}<\infty .
$$

We start the proof of conclusion (3.41) with the formula,

$$
\left[\left(I+Q_{u}\right)^{-1} U(2)\left(I+Q_{u}\right)-\left(I+Q_{u}\right)^{-1} Q_{u}^{\prime}\right]_{12}=-q_{u}^{\prime}+U(2)_{12}+2 U(2)_{11} q_{u} .
$$

To prove this formula, note that the definition (3.40) shows that formula (3.21) also holds for $Q_{u}$,

$$
Q_{u}^{2}=0 \text { and so }\left(I+Q_{u}\right)^{-1}=I-Q_{u} .
$$

This formula allows us to repeat the proof of formula (3.20) and conclude that for each matrix $B$,

$$
\begin{aligned}
& \left(I+Q_{u}\right)^{-1} B\left(I+Q_{u}\right)-\left(I+Q_{u}\right)^{-1} Q_{u}^{\prime}= \\
& {\left[\begin{array}{cc}
B_{11}-q_{u} B_{21} & -q_{u}^{\prime}+B_{12}+q_{u}\left(B_{11}-B_{22}\right)-q_{u}^{2} B_{21} \\
B_{21} & B_{22}+q_{u} B_{21}
\end{array}\right]}
\end{aligned}
$$

Applying formula (3.45) to the matrix $B=U(2)$ and using that according to the definition $(3.36)$ trace $U(2)=0$ and $U(2)_{21}=0$, we find formula $(3.43)$.

We continue the proof of conclusion (3.41) by constructing an approximate solution to the linear differential equation,

$$
-q_{u}^{\prime}+U(2)_{12}+2 U(2)_{11} q_{u}=0
$$

which we obtained by setting the right member of formula (3.43) equal to zero. Inserting the definition (3.36) and formula (3.23) into the linear differential equation (3.46) and using formula (3.25) to split the term $2 U(1)_{11} q_{u}$ into two, we find,

$$
\begin{gathered}
-q_{u}^{\prime}+\sum_{j=1}^{j=3}\left[c_{12}^{+j} f_{j} \cdot \exp \left(i g_{j}\right)+c_{12}^{-j} f_{j} \cdot \exp \left(-i g_{j}\right)\right]+2 i \mu q_{u} \\
+2 \sum_{j=1}^{j=3}\left\{\left[c_{11}^{+j} f_{j} \cdot \exp \left(i g_{j}\right)+c_{11}^{-j} f_{j} \cdot \exp \left(-i g_{j}\right)\right)\right]+ \\
\left.+\left[c_{12}^{+j} f_{j} \cdot \exp \left(i g_{j}\right)+c_{12}^{-j} f_{j} \cdot \exp \left(-i g_{j}\right)\right] q_{\ell}\right\} q_{u}=0
\end{gathered}
$$


We seek an approximate solution to the equation (3.47) in the class of functions of the form,

$$
q_{u}=\sum_{j=1}^{j=3}\left[b^{+j} f_{j} \cdot \exp \left(i g_{j}\right)+b^{-j} f_{j} \cdot \exp \left(-i g_{j}\right)\right]
$$

where, the functions $b^{ \pm j}$ are slowly varying. Inserting the definition (3.48) into the linear differential equation (3.47) and dropping the derivatives of these slowly varying functions and the second and third order terms in the functions $f_{j}$ we find,

$$
\begin{gathered}
\sum_{j=1}^{j=3}\left\{\left[-i b^{+j} g_{j}^{\prime} f_{j} \cdot \exp \left(i g_{j}\right)+i b^{-j} g_{j}^{\prime} f_{j} \cdot \exp \left(-i g_{j}\right)\right]+\right. \\
\left.+\left[c_{12}^{+j} f_{j} \cdot \exp \left(i g_{j}\right)+c_{12}^{-j} f_{j} \cdot \exp \left(-i g_{j}\right)\right]\right\}+ \\
+2 \sum_{j=1}^{j=3}\left[i \mu b^{+j} f_{j} \cdot \exp \left(i g_{j}\right)+i \mu b^{-j} f_{j} \cdot \exp \left(-i g_{j}\right)\right]=0 .
\end{gathered}
$$

Rearranging the terms in equation (3.49) and setting the coefficients of each of the functions $\exp \left(i g_{j}\right)$ and $\exp \left(-i g_{j}\right)$ equal to zero leads to the formula,

$$
b^{ \pm j} f_{j}=-\left(2 i \mu \pm i g_{j}^{\prime}\right)^{-1} f_{j} \cdot c_{12}^{ \pm j} . \quad \text { for } j=1,2,3 .
$$

From now on we shall define these coefficient functions by formula $\left(3.50_{ \pm}\right)$.

We complete the proof of conclusion (3.41) by inserting the definitions (3.48), (3.36) and formula (3.25), (3.23) into the formula (3.43). Then defining,

$$
e_{12}=\left[\left(I+Q_{u}\right)^{-1} U(2)\left(I+Q_{u}\right)-\left(I+Q_{u}\right)^{-1} Q_{u}^{\prime}\right]_{12},
$$

and using the equation (3.49) we find,

$$
\begin{array}{r}
e_{12}=-\sum_{\substack{j=1 \\
j=3}}^{j=3}\left[\left(b^{+j} f_{j}\right)^{\prime} \cdot \exp \left(i g_{j}\right)+\left(b^{-j} f_{j}\right)^{\prime} \cdot \exp \left(-i g_{j}\right)\right] \\
+2 \sum_{j=1}^{j}\left\{\left[c_{11}^{+j} f_{j} \cdot \exp \left(i g_{j}\right)+c_{11}^{-j} f_{j} \cdot \exp \left(-i g_{j}\right)\right)\right]+ \\
\left.\quad\left[c_{12}^{+j} f_{j} \cdot \exp \left(i g_{j}\right)+c_{12}^{-j} f_{j} \cdot \exp \left(-i g_{j}\right)\right] q_{\ell}\right\} q_{u}
\end{array}
$$

Similarly to the way that formula (3.33), assumptions (2.15), (2.10), (2.9), (2.8) and (2.5) and the definitions (3.31 \pm ), (3.27) implied conclusion (3.16), we see that formula (3.52), the definitions $\left(3.50_{ \pm}\right),(3.48)$ and these assumptions imply conclusion (3.41).

At the same time, we see that assumptions (2.15), (2.9) and (2.8) imply conclusion (3.42). Thus the proof of Lemma 3.4 is complete. 
Applying formula (3.45) to the matrix $B=U(2)+R(2)$ and using the definition (3.51) we find,

$$
\begin{gathered}
\left(I+Q_{u}\right)^{-1}(U(2)+R(2))\left(I+Q_{u}\right)-\left(I+Q_{u}\right)^{-1} Q_{u}^{\prime}= \\
{\left[\begin{array}{cc}
(U(2)+R(2))_{11} & e_{12}+R(2)_{12} \\
(U(2)+R(2))_{21} & (U(2)+R(2))_{22}
\end{array}\right]+} \\
+q_{u}\left[\begin{array}{cc}
(U(2)+R(2))_{21} & R(2)_{11}-R(2)_{22}-q_{u} R(2)_{21} \\
0 & -(U(2)+R(2))_{21}
\end{array}\right]
\end{gathered}
$$

Next define,

$$
\begin{gathered}
R(3)=\left[\begin{array}{cc}
R(2)_{11} & e_{12}+R(2)_{12} \\
R(2)_{21} & R(2)_{22}
\end{array}\right]+ \\
+q_{u}\left[\begin{array}{cc}
R(2)_{21} & R(2)_{11}-R(2)_{22}-q_{u} R(2)_{21} \\
R(2)_{21} & R(2)_{22}-R(2)_{21}
\end{array}\right]+q_{\ell}\left[\begin{array}{cc}
U(1)_{12} & 0 \\
0 & U(1)_{12}
\end{array}\right] .
\end{gathered}
$$

and

$$
U(3)=\left[\begin{array}{cc}
U(1)_{11} & 0 \\
0 & -U(1)_{11}
\end{array}\right] .
$$

Combining the definitions (3.55), (3.54) and (3.36) with formula (3.53) we have,

$$
\left(I+Q_{u}\right)^{-1}(U(2)+R(2))\left(I+Q_{u}\right)-\left(I+Q_{u}\right)^{-1} Q_{u}^{\prime}=U(3)+R(3) .
$$

We see from formula (3.56) that the transformation

$$
u_{3}=\left(I+Q_{u}\right)^{-1} u_{2}
$$

carries the system (3.39) into

$$
u_{3}^{\prime}=\left[U(3)+(R(3)] u_{3} .\right.
$$

As a fourth and final step of the proof of Theorem 3.1 we define an approximate fundamental matrix to the basic system (2.21) by,

$$
Y_{r}=Y_{r}(t, \lambda)=A(t)\left(I+Q_{\ell}(t)\right)\left(I+Q_{u}(t)\right) \cdot \exp \left[\int_{c}^{t} U(3)(s) d s\right], c \leq t .
$$

Then we see from the product rule of differentiation that the definition

$$
\begin{aligned}
C_{r} & =A^{\prime} A^{-1}+A Q_{\ell}^{\prime} A^{-1}+A\left(I+Q_{\ell}\right) Q_{u}^{\prime}\left(I+Q_{\ell}\right)^{-1} A^{-1}+ \\
& +A\left(I+Q_{\ell}\right)\left(I+Q_{u}\right) U(3)\left(I+Q_{u}\right)^{-1}\left(I+Q_{\ell}\right)^{-1} A^{-1}
\end{aligned}
$$


implies,

$$
Y_{r}^{\prime}=C_{r} Y_{r}
$$

Next define,

$$
E_{r}=-A\left(I+Q_{\ell}\right)\left(I+Q_{u}\right) R(3)\left(I+Q_{u}\right)^{-1}\left(I+Q_{\ell}\right)^{-1} A^{-1} .
$$

Then combining the definitions (3.62), (3.60) with formula (3.56), (3.37) and (3.12) we find,

$$
C_{r}=V-\lambda J+E_{r}
$$

and so, we see from the system (3.61) and from assumption (2.3) that,

$$
\left.Y_{r}^{\prime}=\left[(V-\lambda J)+E_{r}\right)\right] Y_{r} .
$$

Finally, using the definition (3.59) we define

$$
z_{r}=Y_{r} \cdot\left[\begin{array}{l}
1 \\
0
\end{array}\right]
$$

and

$$
y_{r}=Y_{r} \cdot\left[\begin{array}{l}
0 \\
1
\end{array}\right]
$$

Then, clearly conclusion (3.2) of Theorem 3.1 holds for each of these two vectors.

We start the proof of conclusion (3.1) by showing that,

$$
\sup _{\operatorname{Re} \lambda \in \mathcal{I}}\|R(3)(., \lambda)\|_{\mathcal{L}^{1}(c, \infty)<\infty} .
$$

To verify estimate (3.67) first, we combine the definition (3.35) with conclusions (3.17) and (3.16) of Lemma 3.2 and with estimate (3.14). This yields,

$$
\sup _{\operatorname{Re\lambda \in \mathcal {I}}}\|R(2)(., \lambda)\|_{\mathcal{L}^{1}(c, \infty)}<\infty .
$$

Combining this estimate with conclusions (3.42) and (3.41) of Lemma 3.3 we find,

$$
\sup _{\operatorname{Re} \lambda \in \mathcal{I}}\left\|\left(q_{u} R(2)\right)(., \lambda)\right\|_{\mathcal{L}^{1}(c, \infty)}<\infty .
$$

Second, we claim that

$$
\sup _{\operatorname{Re} \lambda \in \mathcal{I}}\left\|q_{\ell} U(1)_{12}(., \lambda)\right\|_{\mathcal{L}^{1}(c, \infty)}<\infty .
$$


To see estimate (3.68) we need that formulae (3.27) and (3.23) together yield,

$$
\begin{array}{r}
q_{\ell} U(1)_{12}=\sum_{j=1}^{j=3} \sum_{k=1}^{k=3}\left\{\left[a^{+j} f_{j} \cdot \exp \left(i g_{j}\right)+a^{-j} f_{j} \exp \left(-i g_{j}\right)\right] .\right. \\
\left.\cdot\left[c_{12}^{+k} f_{k} \cdot \exp \left(i g_{k}\right)+c_{12}^{-k} f_{k} \cdot \exp \left(-i g_{k}\right)\right]\right\} .
\end{array}
$$

Note that this formula contains only second order terms in the functions $f_{j}$, and so, combining it with estimates (3.29), (3.28) and (3.24) we obtain estimate (3.68). Then, inserting these estimates into the definition (3.54) we arrive at estimate (3.67).

We continue the proof of conclusion (3.1) by combining conclusion (3.42) of Lemma 3.3 with the definition (3.40) and by combining conclusion (3.17) of Lemma 3.2 with the definition (3.15). This yields,

$$
\sup _{\operatorname{Re\lambda \in \mathcal {I}}}\left\|\left(I+Q_{\ell}(., \lambda)\right)\left(I+Q_{u}(., \lambda)\right)\right\|_{\mathcal{L}^{\infty}(c, \infty)}<\infty .
$$

and so, we see from the first half of conclusion (3.8) of Lemma 3.1 that

$$
\sup _{\operatorname{Re} \lambda \in \mathcal{I}} \| A(., \lambda)\left(I+Q_{\ell}(., \lambda)\left(I+Q_{u}(., \lambda)\right) \|_{\mathcal{L}^{\infty}(c, \infty)}<\infty .\right.
$$

Combining estimate (3.69), in turn, with formula (3.44), (3.21) and with the second half of conclusion (3.8) of Lemma 3.1 we find,

$(3.70) \sup _{\operatorname{Re\lambda } \in \mathcal{I}}\left\|\left(I+Q_{u}(., \lambda)\right)^{-1}\left(I+Q_{\ell}(., \lambda)\right)^{-1} A(., \lambda)^{-1}\right\|_{\mathcal{L}^{\infty}(c, \infty)}<\infty$.

Finally, combining estimates (3.70), (3.69) and (3.67) with the definition (3.62) we obtain conclusion (3.1) of Theorem 3.1

To verify conclusion (3.3) we note that it is an immediate consequence of the definitions (3.66) and (3.6). In fact, this property motivated the choice of the branch in the definition (3.6).

4. Estimates for the solutions of the basic system (2.21) near zero. We start this section by describing approximate solutions to the basic system $(2.21)$ on the interval $(0, c)$. For this purpose, first with the help of the definition (2.12) define an approximate system by,

$$
Y_{\ell}^{\prime}(t)=\frac{1}{t} J C_{0}(e, \ell) Y_{\ell}(t)
$$

To motivate this choice, note that by assumptions (2.13) and (2.12) the coefficient matrices of the systems (4.1) and (2.21) differ by a bounded matrix. Since the function values of the coefficient matrix of the system (4.1) commute, it has a fundamental matix of the form,

$$
Y_{\ell}(t)=\exp \left[\int_{c}^{t} \frac{1}{\sigma} J C_{0}(e, \ell) d \sigma\right]
$$


Second, we need the elementary fact that the spectrum of this coefficient matrix is given by,

$$
\operatorname{Spec}\left(J C_{0}(e, \ell)=\left\{-\left(\ell^{2}-e^{2}\right)^{1 / 2},\left(\ell^{2}-e^{2}\right)^{1 / 2}\right\} .\right.
$$

Hence there are vectors $a_{ \pm}$, such that

$$
J C_{0}(e, \ell) a_{ \pm}= \pm\left(\ell^{2}-e^{2}\right)^{1 / 2} a_{ \pm} .
$$

Then, we define the approximate solution $y_{\ell}$ by,

$$
y_{\ell}=Y_{\ell}(t) a_{+},
$$

and the corresponding weight function by

$$
w_{\ell}(t)=\left|y_{\ell}(t)\right| .
$$

With the help of this weight function for each $f$ in $C(0, c)$ we define the norm,

$$
\|f\|_{w_{\ell}}=\sup _{0<t<c}|f(t)| w_{\ell}(t)^{-1},
$$

and denote by $B_{w_{\ell}}$ the space of those functions for which this norm is finite.

In the following theorem we show that the basic system (2.21) also admits a solution which is asymptotic to $y_{\ell}(t, \lambda)$ at $t=0$ and for which this norm is finite.

THEOREM 4.1. Let the assumptions of Theorem 2.1 hold, let the approximate solution $y_{\ell}$ be given by the definition (4.5) and let the space $B_{w_{\ell}}$ be given with the help of the definition (4.7). Then, on the interval $(0, c)$ the basic system (2.21) admits a solution $f_{\ell}$ in $B_{w_{\ell}}$ such that,

$$
\sup _{\operatorname{Re\lambda } \in \mathcal{I}}\left\|f_{\ell}(\cdot, \lambda)\right\|_{w_{\ell}}<\infty
$$

and

$$
\sup _{\operatorname{Re} \lambda \in \mathcal{I}} \lim _{t \rightarrow 0}\left|f_{\ell}(t, \lambda)-y_{\ell}(t, \lambda)\right| \cdot\left|y_{\ell}(t, \lambda)\right|^{-1}=1 .
$$

We start the proof of Theorem 4.1 by deriving a Volterra equation for such a solution of the basic system (2.21). To do this, first define the error potential to be the difference of the coefficient matrices of the original system (2.21) and of the approximate system (4.1),

$$
E_{\ell}(t)=(V(t)-\lambda J)-\frac{1}{t} J C_{0}(e, \ell) .
$$

Then adding $E_{\ell} u$ to both sides of the basic system (2.21) we find,

$$
u^{\prime}-\frac{1}{t} J C_{0}(e, \ell) u=E_{\ell} u \text {. }
$$


Second, with the help of the definitions (4.4) and (4.2) define,

$$
z_{\ell}=Y_{\ell}(t) a_{-} .
$$

Then it is clear from the definitions (4.5) and (4.12) that each of these two approximate solutions satisfies the homogeneous equation corresponding to the system (4.11). Third, with the notation of [LR], define the kernel

(4.13) $Q_{\ell}(t, s)=\left(y_{\ell}(t)><J z_{\ell}(s)-z_{\ell}(t)><J y_{\ell}(s)\right)<y_{\ell}, J z_{\ell}>^{-1} E_{\ell}(s)$

and the corresponding operator

$$
Q_{\ell} f(t)=\int_{0}^{t} Q_{\ell}(t, s) f(s) d s, \quad f \in C_{0}^{\infty}\left((0, c), \mathcal{C}_{2}\right) .
$$

Then, we know that (e.g. [LR]

$$
\left[\left(D-(V-\lambda J)+E_{\ell}\right] Q_{\ell} f=E_{\ell} f, \quad f \in C_{0}^{\infty}\left((0, c), \mathcal{C}_{2}\right) .\right.
$$

We see from the definitions (4.10) and (4.5) that

$$
\left[\left(D-(V-\lambda J)+E_{\ell}\right] y_{\ell}=0,\right.
$$

and so, relation (4.15) shows that each solution $f_{\ell}$ of the Volterra equation

$$
f_{\ell}=y_{\ell}+Q_{\ell} f_{\ell}
$$

also satisfies the basic system (4.11).

We continue the proof of Theorem 4.1 by making essential use of a result of Love [L], Erdelyi [Er] and Olver [O], [RT]. To formulate it, following them we define

$$
\left\|Q_{\ell}\right\|\left(L E O, w_{\ell}\right)=\int_{\mathcal{I}} \sup _{\xi>\eta} w_{\ell}(\xi)^{-1} w_{\ell}(\eta)\left|Q_{\ell}(\xi, \eta)\right| d \eta .
$$

Lemma 4.1. Let the Volterra operator $Q_{\ell}$ be given by the definition (4.14) and let the space $\mathbf{B}_{w_{\ell}}$ be given with the help of the definition (4.7). Assume that

$$
\left\|Q_{\ell}\right\|\left(L E O, w_{\ell}\right)<\infty
$$

Then,

$$
Q_{\ell} \in \mathbf{B}\left(\mathbf{B}_{w_{\ell}}\right) \quad \text { and } \quad\left\|Q_{\ell}\right\|\left(w_{\ell}\right) \leq\left\|Q_{\ell}\right\|\left(L E O, w_{\ell}\right) .
$$

Furthermore, for each $\lambda \neq 0$ in $\mathbf{C}$,

$$
\left(\lambda I-Q_{\ell}\right)^{-1} \in \mathbf{B}\left(\mathbf{B}_{w_{\ell}}\right)
$$

and

$$
\|\left(\lambda I-Q_{\ell}\right)^{-1}||\left(w_{\ell}\right) \leq|\lambda|^{-1} \exp \left(|\lambda|^{-1}\left\|Q_{\ell}\right\|\left(L E O, w_{\ell}\right)\right) .
$$

We complete the proof of Theorem 4.1 by noting that application of the LEO Lemma 4.1 to the Volterra operator of the definition (4.14) yields Theorem 4.1. 
5. Estimates for the solutions of the basic system (2.21) near infinity. We start this section by defining a weight function with the help of the approximate solution of the definition (3.66). More specifically, we define,

$$
w_{r}(t)=\left|y_{r}(t)\right|
$$

Then, with the help of this weight function for each $f$ in $C(c, \infty)$ define the norm,

$$
\|f\|_{w_{r}}=\sup _{t>c}|f(t)| w_{r}(t)^{-1}
$$

and denote by $B\left(w_{r}\right)$ the space of those functions for which this norm is finite. In the following theorem we show that the basic system (2.21) admits a solution which is asymptotic to $y_{r}(t, \lambda)$ at $t=\infty$ and for which this norm is finite.

THEOREM 5.1. Let the assumptions of Theorem 2.1 hold, let the approximate solution $y_{r}$ be given by the definition (3.66) and let the space $\mathbf{B}_{r}$ be given with the help of the definition (5.2). Then, the basic system (2.21) admits a solution $f_{r}$ in $\mathbf{B}_{r}$ such that,

$$
\sup _{\operatorname{Re} \lambda \in \mathcal{I}}\|f(\cdot, \lambda)\|_{w_{r}}<\infty
$$

and

$$
\sup _{\operatorname{Re} \lambda \in \mathcal{I}} \lim _{t \rightarrow \infty}\left|f_{r}(t, \lambda)-y_{r}(t, \lambda)\right| \cdot\left|y_{r}(t, \lambda)\right|^{-1}=1
$$

We start the proof of conclusion (5.3) by deriving Volterra equations for the solutions of the basic system (2.21). To do this, first note that adding an arbitrary function $E_{r} u$ to both sides of the basic system $(2.21)$ we find,

$$
u^{\prime}-(V-\lambda J) u+E_{r} u=E_{r} u .
$$

Second, we choose this arbitrary function by the definition (3.62) and define the kernel

$$
Q_{r}(t, s)=\left(y_{r}(t)><J z_{r}(s)-z_{r}(t)><J y_{r}(s)\right)<y_{r}(s), J z_{r}(s)>^{-1} E_{r}(s)
$$

and the corresponding operator

$$
Q_{r} f(t)=\int_{t}^{\infty} Q_{r}(t, s) f(s) d s, \quad f \in C_{0}^{\infty}\left((c, \infty), \mathcal{C}_{2}\right)
$$

Then, it is not difficult to show that

$$
\left[\left(D-(V-\lambda J)+E_{r}\right] Q_{r} f=E_{r} f, \quad f \in C_{0}^{\infty}\left((c, \infty), \mathcal{C}_{2}\right) .\right.
$$


We see from conclusion (3.2) of Theorem 3.1 that

$$
\left[\left(D-(V-\lambda J)+E_{r}\right] y_{r}=0\right.
$$

and so, relation (5.8) shows that each solution $f_{r}$ of the Volterra equation

$$
f_{r}=y_{r}+Q_{r} f_{r}
$$

also satisfies the system (5.5).

We continue the proof of conclusion (5.3) by showing that the Volterra operator of the definition (5.7) satisfies the assumptions of the LEO Lemma 4.1, which is the statement of the lemma that follows. Here of course, we use $Q_{r}$ in place of $Q_{\ell}$ and $w_{r}$ in place of $w_{\ell}$. For completeness, we display the definition (4.18) with these two replacements,

$$
\left\|Q_{r}\right\|\left(L E O, w_{r}\right)=\int_{\mathcal{I}} \sup _{\xi>\eta} w_{r}(\xi)^{-1} w_{r}(\eta)\left|Q_{r}(\xi, \eta)\right| d \eta .
$$

LEMMA 5.1. Let the Volterra operator $Q_{r}$ be given by the definition (5.7) and let the space $\mathbf{B}\left(w_{r}\right)$ be given with the help of the definitions (5.2) and (3.66). Then,

$$
\sup _{\operatorname{Re\lambda \in \mathcal {I}}}\left\|Q_{r}\right\|\left(L E O, w_{r}\right)<\infty .
$$

We start the proof of Lemma 5.1 by showing that it holds for the first term of formula (5.6);

$$
\begin{aligned}
\int_{c}^{\infty} & \sup _{t>s} w_{r}(t)^{-1} w_{r}(s) \mid y_{r}(t)> \\
& <J z_{r}(s)|\cdot|<y_{r}(s), J z_{r}(s)>^{-1}|\cdot| E_{r}(s) \mid d s<\infty .
\end{aligned}
$$

To prove estimate (5.13), first we show that,

$$
\sup _{s>c} w_{r}(s)\left|z_{r}(s)\right|<\infty .
$$

To see estimate (5.14) note that according to the definition (3.55) the matrix $U(3)$ is diagonal and trace $U(3)=0$. Hence the definition (3.66) yields,

$$
y_{r}(t)=A(t)\left(I+Q_{\ell}(t)\right)\left(I+Q_{u}(t)\right) \cdot \exp \left[\int_{c}^{t} U(3)_{22}(\sigma) d \sigma\right] \cdot\left[\begin{array}{l}
0 \\
1
\end{array}\right]
$$

and the definition (3.65) yields,

$$
(5.16) z_{r}(s)=A(s)\left(I+Q_{\ell}(s)\right)\left(I+Q_{u}(s)\right) \cdot \exp \left[-\int_{c}^{s} U(3)_{22}(\sigma) d \sigma\right] \cdot\left[\begin{array}{l}
1 \\
0
\end{array}\right]
$$

Taking the absolute values of formula (5.16) and (5.15) and multiplying the resulting formula together we find,

$$
\left|y_{r}(s)\right| \cdot\left|z_{r}(s)\right| \leq\left\|A(s)\left(I+Q_{\ell}(s)\right)\left(I+Q_{u}(s)\right)\right\|^{2} .
$$


Combining estimate (5.17) with the definition (5.1) and with estimate (3.69) we find estimate (5.14). To prove estimate (5.13), second we show that,

$$
\inf _{\operatorname{Re} \in \in \mathcal{I}} \inf _{s>c}\left|<y_{r}(s), J z_{r}(s)>\right| \neq 0 .
$$

To see estimate (5.18) note that formula (5.16) and (5.15) together yield,

$$
\begin{aligned}
& <y_{r}(s), J z_{r}(s)>=\left\{<A(s)\left(I+Q_{\ell}(s)\right)\left(I+Q_{u}(s)\right)\left[\begin{array}{l}
1 \\
0
\end{array}\right],\right. \\
& \left.J A(s)\left(I+Q_{\ell}(s)\right)\left(I+Q_{u}(s)\right)\left[\begin{array}{l}
0 \\
1
\end{array}\right]>\right\} .
\end{aligned}
$$

Since an invertible matrix maps linearly independent vectors into linearly independent vectors, we see from this formula and from estimate (3.70) that estimate (5.18) holds. Finally, we note that combining estimates (5.18) and (5.14) with conclusion (3.1) of Theorem 3.1 and with the definition (5.1) we find estimate (5.13).

We continue the proof of Lemma 5.1 by showing that it also holds for the second term of formula (5.6);

$$
\begin{aligned}
\int_{c}^{\infty} & \sup _{t>s} w_{r}(t)^{-1} w_{r}(s)\left|z_{r}(t)><J y_{r}(s)\right| . \\
& \cdot\left|<y_{r}(s), J z_{r}(s)>^{-1}\right| \cdot\left|E_{r}(s)\right| d s<\infty .
\end{aligned}
$$

As a first step of the proof of estimate (5.19) we show the existence of a constant $\gamma$, such that,

$$
w_{r}(s) \cdot\left|y_{r}(s)\right| \leq \gamma \cdot \exp \left[-\operatorname{Re} 2 \int_{c}^{s} i \mu(\sigma) d \sigma\right]
$$

To see this, we note that similarly to estimate (5.17),

$$
w_{r}(s) \cdot\left|y_{r}(s)\right| \leq\left\|A(s)\left(I+Q_{\ell}(s)\right)\left(I+Q_{u}(s)\right)\right\|^{2} \cdot \exp \left[\operatorname{Re} 2 \int_{c}^{s} U(3)_{22}(\sigma) d \sigma\right] .
$$

Inserting estimate (3.69) into this one we find a constant $\gamma$ such that

$$
w_{r}(s) \cdot\left|y_{r}(s)\right| \leq \gamma \cdot \exp \left[\operatorname{Re} 2 \int_{c}^{s} U(3)_{22}(\sigma) d \sigma\right]
$$

Next we claim that there is a possibly different constant $\gamma$ such that,

$$
\exp \left[\operatorname{Re} 2 \int_{c}^{s} U(3)_{22}(\sigma) d \sigma\right] \leq \gamma \cdot \exp \left[-\operatorname{Re} 2 \int_{c}^{s} i \mu(\sigma) d \sigma\right] .
$$

To prove estimate (5.23) note that the definition (3.55) and formula (3.25) together yield, 


$$
\begin{aligned}
& \exp \left[2 \int_{c}^{s} U(3)_{22}(\sigma) d \sigma\right]=\exp \left[-2 \int_{c}^{s} i \mu(\sigma) d \sigma\right] \\
& \cdot \exp \left[2 \int_{c}^{s} \sum_{j=1}^{j=3}\left[c_{11}^{+j} f_{j} \cdot \exp \left(i g_{j}\right)+c_{11}^{-j} \cdot f_{j} \exp \left(-i g_{j}\right)\right](\sigma) d \sigma\right]
\end{aligned}
$$

An integration by parts together with estimate (3.24) and assumptions (2.9) and (2.8) shows that the supremum of the absolute value of the second factor is finite. Hence, denoting this supremum by $\gamma$ and taking the absolute value of this formula we find estimate (5.23).

As a second step of the proof of estimate (5.19) we show that there is a constant $\gamma$ such that

$$
w_{r}(t)^{-1} \cdot\left|z_{r}(t)\right| \leq \gamma \exp \left[\operatorname{Re} 2 \int_{c}^{t} i \mu(\sigma) d \sigma\right] .
$$

In fact, combining the definition (5.1) with formula (5.15), (5.16) we find that the constant $\gamma$ of estimate (3.69) is such that

$$
w_{r}(t)^{-1} \cdot\left|z_{r}(t)\right| \leq \gamma \exp \left[-\operatorname{Re} 2 \int_{c}^{t} U(3)_{22}(\sigma) d \sigma\right]
$$

The proof of estimate (5.23) also shows that

$$
\exp \left[-\operatorname{Re} 2 \int_{c}^{s} U(3)_{22}(\sigma) d \sigma\right] \leq \gamma \cdot \exp \left[\operatorname{Re} 2 \int_{c}^{s} i \mu(\sigma) d \sigma\right]
$$

Now combining estimate (5.25) with the previous one, we find estimate (5.24).

As a third and final step of the proof of estimate (5.19) we multiply estimates (5.24) and (5.20) together. This yields,

$$
w_{r}(t)^{-1} \cdot\left|z_{r}(t)\right| w_{r}(s) \cdot\left|y_{r}(s)\right| \leq \gamma^{2} \exp \left[\operatorname{Re} 2 \int_{s}^{t} i \mu(\sigma) d \sigma\right]
$$

Now we need that according to the definition (3.6) the integrand on the right of estimate (5.26) is negative. In fact, this property motivated the choice of the branch of the square root function in the definition (3.5). Hence the exponential in estimate $(5.26)$ is majorized by 1 , and so,

$$
w_{r}(t)^{-1} \cdot\left|z_{r}(t)\right| w_{r}(s) \cdot\left|y_{r}(s)\right| \leq \gamma^{2}
$$

Combining estimates (5.27) and (5.18) with conclusion (3.1) of Theorem 3.1 we obtain estimate $(5.19)$.

We complete the proof of Lemma 5.1 by noting that inserting estimates (5.19) and (5.13) and the definition (5.6) into the definition (5.11) we find conclusion (5.12). 
We complete the proof of conclusion (5.3) by noting that Lemma 5.1 allows us to apply the LEO Lemma 4.2 to the Volterra operator of the definition (5.7) and to the weight function of the definition (5.1). Then conclusion (4.21) with $\lambda=1$, yields the invertibility of the Volterra equation (5.10) and conclusion (4.21) yields,

$$
\|f(\cdot, \lambda)\|_{w_{r}} \leq \exp \left(\left\|Q_{r}\right\|\left(L E O, w_{r}\right)\right) \cdot\left\|y_{r}\right\|_{w_{r}} .
$$

We see from the definitions (5.2) and (5.1) that $\left\|y_{r}\right\|_{w_{r}} \leq 1$ and so, combining estimate (5.28) with conclusion (5.12) of Lemma 5.1, we obtain conclusion (5.3).

To prove conclusion (5.3) note that the already established conclusion (5.3) allows us to conclude that

$$
\lim _{t \rightarrow \infty}\left|Q_{r} f_{r}(t)\right| \cdot\left|y_{r}(t)\right|^{-1}=0
$$

Then combining this estimate with the Volterra equation (5.10) we obtain conclusion (5.4). This completes the proof of Theorem 5.1.

6. Proof of the main theorem 2.1 under the additional assumption (6.3). In this section we prove the main Theorem 2.1 under the additional assumption (6.3), to be stated. The considerations of [DR] show that it is implied by the following Theorem 6.1. In it, for a given angle $0<\theta \leq \pi$ we define

$$
\mathcal{R}_{ \pm}(\mathcal{I})=\{\lambda \in C: \mid \operatorname{Re} \lambda \in \mathcal{I}, 0< \pm \arg \lambda<\theta\}
$$

THEOREM 6.1. Let the assumptions and notations of the main Theorem 2.1 hold. Then, there are regions of the form (6.1) such that,

$$
\sup _{\lambda \in \mathcal{R}_{ \pm}(\mathcal{I})}\left\|m_{s}^{1 / 2} R(\lambda) m_{s}^{1 / 2}\right\|<\infty, s>1 / 2 .
$$

To prove Theorem 6.1 first we need the Weyl-Weidmann construction [We], [DS] for the resolvent kernel of the operator of the basic system (2.21). To describe this construction we make the additional assumption that the constant $e$ of the definition (2.12) is such that,

$$
|e|<\left(\ell^{2}-1 / 4\right)^{1 / 2}
$$

and denote by $f_{r}$ and $f_{\ell}$ the solutions of Theorem 5.1 and of Theorem 4.1, respectively, extended to all of $\mathbf{R}^{+}$. Then according to this construction, in the notation of [LR] this resolvent kernel is given by;

$$
R(\lambda)(\xi, \eta)=<f_{\ell}, J f_{r}>^{-1} \cdot \begin{cases}f_{r}(\xi, \lambda)><f_{\ell}(\eta, \lambda), & \text { for } \eta<\xi \\ f_{r}(\eta, \lambda)><f_{\ell}(\xi, \lambda), & \text { for } \eta>\xi\end{cases}
$$

To prove Theorem 6.1 second we need the Schur-Holmgren-Carleman bound of a given integral operator $\mathrm{R}$ with reference to a given positive 
measurable function $t,[\mathrm{~F}][\mathrm{Ok}]$. This is defined by,

$\|R\|(t)=\left(\sup t(\xi)^{-1} \int_{\mathcal{I}}\|R(\xi, \eta)\| t(\eta) d \eta \cdot \sup t(\eta)^{-1} \int_{\mathcal{I}}\|R(\xi, \eta)\| t(\xi) d \xi\right)^{-1 / 2}$ (6.5)

where the supremum is taken over the support of $t$. According to their result $[\mathrm{F}],[\mathrm{Ok}]$, if the support of $t(\xi) t(\eta)$ contains the support of $R(\xi, \eta)$, then

$$
\|R\| \leq\|R\|(t))
$$

where the left member is the operator norm. In view of the bound (6.6) Theorem 6.1 is implied by the following theorem.

THEOREM 6.2. Let the assumptions of Theorem 6.1 hold, let the real number e satisfy assumption (6.3) and let the function $t$ be given by,

$$
t(\eta)=\eta^{-1 / 2} \text {. }
$$

Then, there are regions of the form (6.1) such that,

$$
\sup _{\lambda \in \mathcal{R}_{ \pm}(\mathcal{I})}\left\|m_{s}^{1 / 2} R(\lambda) m_{s}^{1 / 2}\right\|(t)<\infty .
$$

In the following lemma we isolate the key estimates that we need to prove Theorem 6.2. In it, we extend the weight function of the definition (4.6) to all of $\mathbf{R}^{+}$. More specifically, with the help of the approximate solutions of the definitions (4.5) and (3.65) we define,

$$
w_{\ell, e}(t)=\left\{\begin{array}{ll}
\left|y_{\ell}(t)\right|\left|z_{r}(c)\right|, & \text { for } t \in(0, c) \\
\left|z_{r}(t)\right|\left|y_{\ell}(c)\right|, & \text { for } t \in(c, \infty)
\end{array} .\right.
$$

Similarly, we extend the weight function of the definition (5.1) to all of $\mathbf{R}^{+}$,

$$
w_{r, e}(t)=\left\{\begin{array}{ll}
\left|z_{\ell}(t)\right|\left|y_{r}(c)\right|, & \text { for } t \in(0, c) \\
\left|y_{r}(t)\right|\left|z_{\ell}(c)\right|, & \text { for } t \in(c, \infty)
\end{array} .\right.
$$

LEMMA 6.1. Let the assumptions of the main Theorem 2.1 hold and let the $f_{\ell}$ and $f_{r}$ be the solutions of Theorems 4.1 and 5.1 respectively. Then, the weight functions of the definitions (6.9) and (6.10) are such that,

$$
\sup _{\operatorname{Re\lambda \in \mathcal {I}}}\left\|f_{\ell}(\cdot, \lambda)\right\|_{w_{\ell, e}}<\infty
$$

and

$$
\sup _{\operatorname{Re} \lambda \in \mathcal{I}}\left\|f_{r}(\cdot, \lambda)\right\|_{w_{r, \epsilon}}<\infty \text {. }
$$

Furthermore, the boundary value of their Wronskian, as $\lambda+i \epsilon \rightarrow \lambda \in \mathcal{I}$ from above, is such that,

$$
\inf _{\lambda \in \mathcal{I}}\left|<f_{\ell}(\lambda), J f_{r}(\lambda)>\right| \neq 0
$$


As a first step of the proof of conclusion (6.11) define the weight function

$$
w_{r, 1}(t)=\left|z_{r}(t)\right|, \quad \text { for } t \in(c, \infty)
$$

Then, similarly to the proof of Theorem 5.1 we see that the basic system (2.21) admits a solution $g_{r}$ such that,

$$
\sup _{\operatorname{Re} \in \mathcal{L}}\left\|g_{r}(\cdot, \lambda)\right\|_{w_{r, 1}}<\infty
$$

and

$$
\sup _{\operatorname{Re\lambda } \in \mathcal{I}} \lim _{t \rightarrow \infty}\left(\left|g_{r}(t, \lambda)-z_{r}(t, \lambda)\right| \cdot\left|z_{r}(t, \lambda)\right|^{-1}\right)=1
$$

The asymptotic formula (6.16), conclusion (5.4) of Theorem 5.1 and the definitions (3.66), (3.65) together show that the solutions $f_{r}$ and $g_{r}$ are linearly independent. Hence there are constants $\alpha_{r}(\lambda)$ and $\beta_{r}(\lambda)$, such that

$$
f_{\ell}(t, \lambda)=\alpha_{r}(\lambda) f_{r}(t, \lambda)+\beta_{r}(\lambda) g_{r}(t, \lambda) \quad \text { for } t \in(c, \infty)
$$

As a second step of the proof of conclusion (6.11) we show that

$$
\sup _{\operatorname{Re} \lambda \in \mathcal{I}}\left|\alpha_{r}(\lambda)\right|<\infty
$$

To prove estimate (6.18) first note that formula (6.17) yields,

$$
\alpha_{r}(\lambda)=<f_{\ell}(c, \lambda), J g_{r}(c, \lambda)>\cdot\left(<f_{r}(c, \lambda), J g_{r}(c, \lambda)>\right)^{-1}
$$

Combining conclusion (4.8) of Theorem 4.1 with the definition (4.6) and combining estimate (6.15) with the definition (6.14), we see that there is a constant $\gamma$ such that,

$$
\left|<f_{\ell}(c, \lambda), J g_{r}(c, \lambda)>\right| \leq \gamma\left|y_{\ell}(c, t)\right|\left|z_{r}(c, t)\right|
$$

Combining this estimate, in turn, with the definitions (4.5), (3.65) and with estimate (3.69) we find,

$$
\sup _{\operatorname{Re} \lambda \in \mathcal{I}}\left|<f_{\ell}(c, \lambda), J g_{r}(c, \lambda)>\right|<\infty
$$

In other words, estimate (6.18) holds for the first factor of formula (6.19). To see that it also holds for the second factor we need that the trace of the coefficient matrix of the basic system (2.21) is 0 , and so,

$$
<f_{r}(c, \lambda), J g_{r}(c, \lambda)>=\lim _{t \rightarrow \infty}<f_{r}(t, \lambda), J g_{r}(t, \lambda)>
$$


Conclusion (5.4) of Theorem 5.1 and the asymptotic formula (6.16) together show that

$$
\left.\lim _{t \rightarrow \infty}<f_{r}(t, \lambda), J g_{r}(t, \lambda)>=\lim _{t \rightarrow \infty}<y_{r}(t, \lambda), J z_{r}(t, \lambda)\right\rangle .
$$

Combining this formula with estimate (5.18) we find,

$$
\inf _{R \in \lambda \in \mathcal{I}} \mid\left(<f_{r}(c, \lambda), J g_{r}(c, \lambda)>\right) \neq 0
$$

Hence estimate (6.18) holds for the second factor of formula (6.19), and this completes the proof of estimate (6.18).

As a third step of the proof of conclusion (6.11) we show that

$$
\sup _{\operatorname{Re} \in \mathcal{I}}\left|\beta_{r}(\lambda)\right|<\infty .
$$

To prove estimate (6.23) first note that formula (6.17) yields,

$$
\beta_{r}(\lambda)=<f_{\ell}(c, \lambda), J f_{r}(c, \lambda)>\cdot\left(<g_{r}(c, \lambda), J f_{r}(c, \lambda),>\right)^{-1}
$$

Similarly to the way that conclusion (4.8) of Theorem 4.1 did imply estimate (6.21), we see that conclusion (5.3) of Theorem 5.1 implies,

$$
\sup _{\operatorname{Re} \lambda \in \mathcal{I}}\left|<f_{\ell}(c, \lambda), J f_{r}(c, \lambda)>\right|<\infty \text {. }
$$

Inserting estimates (6.25) and (6.22) into formula (6.24) we find estimate $(6.23)$.

As a fourth step of the proof of conclusion (6.11) we note that combination of estimates (5.25) and (5.23) with formula (5.16) and (5.15) and with the definition (3.6) yields,

$$
\left|y_{r}(t)\right| \leq \gamma \cdot\left|z_{r}(t)\right|, \quad \text { for } t \in(c, \infty)
$$

Combining estimate (6.26), in turn, with conclusion (5.3) of Theorem 5.1 and with the definition (6.14) we find,

$$
\sup _{\operatorname{Re\lambda \in \mathcal {I}}}\left\|f_{r}(\cdot, \lambda)\right\|_{w r, 1}<\infty
$$

Inserting estimates $(6.27),(6.23),(6.18)$ and (6.15) into formula (6.17) we obtain,

$$
\sup _{\operatorname{Re\lambda } \in \mathcal{I}}\left\|f_{\ell}(\cdot, \lambda)\right\|_{w r, 1}<\infty
$$

Finally, combining estimate (6.28) with conclusion (4.8) of Theorem 4.1 and with the definition (6.9), we arrive at conclusion (6.11).

The proof of conclusion (6.12) is similar and for brevity we skip the details. 
We start the proof of conclusion (6.13) by showing that for large enough $t$

$$
f_{r 1}(t) \neq 0 \quad \text { and } \quad f_{r_{2}}(t) \neq 0 .
$$

Here, of course the subscripts 1,2 denote the components of this vector. We see from assumption (2.14) that to show relation (6.29) it suffices to show that

$$
\lim _{t \rightarrow \infty} f_{r_{2}}(t) f_{r 1}(t)^{-1}=(i \mu(\infty)+m) \cdot \lambda^{-1}
$$

To see relation (6.30), we need that the definitions (3.66), (3.59) and (3.55) together with conclusions (3.42) and (3.17) of Lemmata 3.3 and 3.2 yield,

$$
y_{r}(t) \sim\left(-\exp \int_{e}^{t} U(1)_{11}(\sigma) d \sigma\right) \cdot A(\infty)\left[\begin{array}{l}
0 \\
1
\end{array}\right], \quad \text { for } t \rightarrow \infty
$$

We see from conclusion (3.7) of Lemma 3.1 that the vector on the right is an eigenvector of the matrix $P(\infty)-\lambda J$ with eigenvalue $-i \mu(\infty)$. This fact, assumption (2.6) and the asymptotic formula (6.31) together allow us to verify the asymptotic formula $(6.30)$ for the approximate solution $y_{r}$. Combining this asymptotic formula with conclusion (5.4) of Theorem 5.1 we find the asymptotic formula $(6.30)$ for the solution $f_{r}$ itself.

We continue the proof of conclusion (6.13) by showing that if $t$ is so large that relation (6.29) holds, then

$$
\begin{aligned}
& \operatorname{Im}\left\{<f_{\ell}(\lambda), J f_{r}(\lambda)>f_{\ell 1}(t)^{-1} f_{r 1}(t)^{-1}\right\}=\operatorname{Im}\left\{f_{r 2}(t) f_{r 1}(t)^{-1}\right\} \\
& \quad \text { for } f_{\ell 1}(t)^{-1} \neq 0
\end{aligned}
$$

and

$$
\begin{aligned}
& \operatorname{Im}\left\{<f_{\ell}(\lambda), J f_{r}(\lambda)>f_{\ell 2}(t)^{-1} f_{r 21}(t)^{-1}\right\}=\operatorname{Im}\left\{f_{r 1}(t) f_{r 2}(t)^{-1}\right\} \\
& \quad \text { for } f_{\ell 2}(t)^{-1} \neq 0,
\end{aligned}
$$

For brevity we verify formula (6.32) only. The definition of $J$ yields,

$$
\begin{aligned}
& <f_{\ell}(\lambda), J f_{r}(\lambda)>f_{\ell 1}(t)^{-1} f_{r}(t)^{-1}=f_{r_{2}}(t) f_{r 1}(t)^{-1}-f_{\ell 2}(t) f_{\ell 1}(t)^{-1} \\
& \quad \text { for } f_{\ell 1}(t) \neq 0
\end{aligned}
$$

We see from the additional assumption (6.3) that,

$$
\operatorname{Im}\left(\ell^{2}-e^{2}\right)^{1 / 2}=0
$$

and so, by formula (4.3) the eigenvalues of the matrix $J C(e, \ell)$ are real. Since according to the definition (2.12) this matrix is real, it follows that the eigenvectors are also real. Combining these two facts with the definition (4.5) we find that the approximate solution $y_{\ell}$ is real;

$$
\operatorname{Im} y_{\ell}=0 \text {. }
$$


Combining this relation, in turn, with conclusion (4.9) of Theorem 4.1 and with the fact that the coefficients of the basic system $(2.21)$ are real, we obtain that the solution $f_{\ell}$ is also real;

$$
\operatorname{Im} f_{\ell}=0 \text {. }
$$

Inserting formula (6.37) into formula (6.34) we arrive at formula (6.32). We see from the uniqueness of the Cauchy problem of the basic system (2.21) and from conclusion (4.9) of Theorem 4.1 and the definition (4.5) that for each $t, f_{r}(t) \neq 0$. Hence one of the components of this vector is not 0 , and so either formula (6.32) or formula (6.33) holds. Combining this fact with the the asymptotic formula (6.30) and with assumption (2.14) we obtain,

$$
<f_{\ell}(\lambda), J f_{r}(\lambda)>\neq 0
$$

We see from Theorems 4.1 and 5.1 that the left member of relation (6.38) depends continuously on $\lambda \in \mathcal{I}$ and so, conclusion (6.13) follows. This completes the proof of Lemma 6.2.

Now it is straightforward to show that Lemma 6.2 implies Theorem 6.2. Since in Lemma 5.1 of [LR] we proved a similar implication, we omit the details and consider the proof of Theorem 6.2 complete. This also completes the proof of the main Theorem 2.1

7. Removal of the additional assumption 6.3. In this section we remove the additional assumption (6.3). Accordingly, let

$$
|e| \geq\left(\ell^{2}-1 / 4\right)^{1 / 2} \text {. }
$$

Then the closure of the formal operator of the definition (2.1) is no longer self-adjoint and the Weyl-Weidmann construction leading to formula (6.4) breaks down. However, we can use this construction for the resolvent of a given self-adjoint extension of this formal operator.

To describe this construction we need that assumption (7.1) yields, $\left|\operatorname{Re}\left(\ell^{2}-e^{2}\right)^{1 / 2}\right|<1 / 2$ and so, the definitions (4.12), (4.4) and (4.2) together show that,

$$
y_{\ell} \in \mathcal{L}^{2}(0, c) \text { and } z_{\ell} \in \mathcal{L}^{2}(0, c)
$$

For brevity we only consider the case of,

$$
|e|<\ell
$$

since the other case is quite similar. In this case relation (6.35) is still valid and hence so is formula (6.36). Replacing the definition (4.5) by the definition (4.12) in the proof of formula (6.36) we find,

$$
\operatorname{Im} z_{\ell}=0
$$


Hence, each linear combination with real coefficients of these two approximate solutions is also real. To be specific let $\alpha \in \mathcal{R}$ be given and define,

$$
y_{\ell, \alpha}=\sin \alpha \cdot y_{\ell}+\cos \alpha \cdot z_{\ell} .
$$

Replacing the approximate solution $y_{\ell}$ by $y_{\ell, \alpha}$ in the proof of Theorem 4.1 we see that the basic system (2.21) admits a solution $f_{\ell, \alpha}$ for which conclusions (4.8) and (4.9) hold. Since $y_{\ell, \alpha}$ satisfies a real boundary condition at zero, it follows from conclusion (4.9) that so does $f_{\ell, \alpha}$. Similarly, it follows that we can also replace $f_{\ell}$ by $f_{\ell, \alpha}$ in conclusion (6.13) of Lemma 6.3. Combining these two facts we see that we can make the same replacement in the Weyl-Weidmann formula (6.4). This yields;

$$
R_{\alpha}(\lambda)(\xi, \eta)=<f_{\ell, \alpha}(\lambda), J f_{r}(\lambda)>^{-1} \cdot\left\{\begin{array}{ll}
f_{r}(\xi, \lambda)><f_{\ell, \alpha}(\eta, \lambda), & \text { for } \eta<\xi \\
f_{r}(\eta, \lambda)><f_{\ell, \alpha}(\xi, \lambda), & \text { for } \eta>\xi
\end{array} .\right.
$$

Then we know [We] that the corresponding operator is the resolvent of the self-adjoint extension of the formal operator (2.2) given by the boundary condition of $f_{\ell, \alpha}(\lambda)$. We denote this resolvent operator by $R_{\alpha}(\lambda)$. Similarly, it follows that we can also replace $f_{\ell}$ by $f_{\ell, \alpha}$ in conclusion (6.11) of Lemma 6.2. Thus, we can replace $f_{\ell}$ by $f_{\ell, \alpha}$ in each of the two conclusions of Lemma 6.2. This fact allows us to repeat the proof of Theorem 6.1 under assumptions (7.1) and (7.3) and conclude that

$$
\sup _{\lambda \in \mathcal{R}_{ \pm}(\mathcal{I})}\left\|m_{s}^{1 / 2} R_{\alpha}(\lambda) m_{s}^{1 / 2}\right\|<\infty, \quad s>1 / 2
$$

Since we have seen in Section 6 that Theorem 6.1 implies the main Theorem 2.1, estimate (7.7) yields the Main Theorem 2.1 under assumptions (7.1) and $(7.3)$.

\section{REFERENCES}

[A] AtKinson, F. V. , The asymptotic solution of second-order differential equations, Ann. Mat. Pura-Appl. 37 (1954), pp. 347-378; Oberwolfach Tagungsbericht 20.1-26.1, 1991.

[B] BeHNCKe, HoRst, Absolute continuity of Hamiltonians with von Neumann Wigner potentials, Proc. Amer. Math. Soc. 111 (1991), pp. 373-384; Absolute continuity of Hamiltonians with von Neumann Wigner potentials II, Manuscripta Math. 71 (1991), pp. 163-181.

[BR] Behncke, H. AND ReJTo, P., Schrödinger and Dirac operators with oscillating potentials, Univ. of Minnesota Math. Report \#88-111, 1988; Schrödinger and Dirac operators with von Neumann Wigner type potentials, Preprint, Universität Osnabrück, 1990.

[BD] Ben-Artzi, Matania and Devinatz, Allen, " The limiting absorption principle for partial differential operators," American Mathematical Society, Memoir; Volume 66-Number 364 (1987).

[DMR] Devinatz, Allen, Moeckel, Richard, and Rejto, Peter, A limiting absorption principle for Schrödinger operators with von Neumann-Wigner type potentials, Integral Equations Operator Theory 14 (1991), pp. 13-68. 
[DR] Devinatz, Allen and Rejto, Peter, A limiting absorption principle for Schrödinger operators with oscillating potentials I, J. Diff. Equations $\mathbf{4 9}$ (1983), pp. 29-84; II, J. Diff. Equations 49 (1983), pp. 85-104; Some absolutely continuous ordinary differential operators with oscillating potentials, in "Qualitative properties of differential equations," University of Alberta Press (1987), pp. 95-106. Proceedings of the 1984 Edmonton Conference. (Ed) Allegretto, W. and Butler, G.J.

[DS] Dunford, Nelson and Schwartz, JaCob T., "Linear Operators," WileyInterscience, New York, London (1962) Part II.

[Ea] Eastham, M. S. P., "Asymptotic Solutions of Linear Differential Systems: Application of Levinson theorem," Oxford, Clarendon Press, (1989).

[Ei] EIdus, D. M., The principle of limiting absorption, (in Russian); Amer. Math.Soc.Transl. 47 (2) (1965), pp. 157-191. See the proof of Theorem 4, Mat. Sb. 57 (1962), pp. 1-99.

[En] Enz, C.P., (Ed), "Pauli Lectures on Physics, Vol.5 Wave Mechanics," MIT Press, Cambridge, MA, 1977. See Section 27, The WKB-method.

[Er] ERdeLY, A., Asymptotic solutions of differential equations with transition points or singularities, See Section 4, J. Math. Phys. 1 (1960), pp. 1626.

[F] Friedrichs, K. O., "Spectral theory of operators in Hilbert space," SpringerVerlag, Lecture Notes in Applied Mathematical Sciences, Vol 9, 1973. See Section 20.

[GP] Gilbert, D. J. and Pearson, D. B., On subordinacy and analysis of the spectrum of one-dimensional Schrödinger operators, J. Math. Anal. Appl. 128, (1987), pp. 30-56.

[H] HeINZ, E., Über das absolut stetige Spektrum singulärer Differentialgleichungssysteme, Nr 1, Nachr. Akad. Wiss. Göttingen II. Math-Phys. K1 (1982), pp. 1-9.

[HKS] Hinton, D. B., Klaus, M., Shaw, J. K., Tichmarsh-Weyl-Levinson theory for a four-dimensional Hamiltonian system, Proc. London Math. Soc. 59, (1989), pp. 339-372; Embedded half-bound states for potentials of Wignervon Neumann type, Proc. London Math. Soc. 62 (1991),pp. 607-646.

[HL] Harris, W.A. AND LUTz, D.A., Asymptotic integration of adiabatic oscillators, J. Math. Anal. Appl. 51 (1975), pp. 76-93; A unified theory of asymptotic integration, J. Math. Anal. Appl. 57 (1977), pp. 571-586.

[HS] Hinton, D. B. And SHaw, J. K., Absolutely continuous spectra of Dirac systems with long range, short range and oscillating potentials, Quart. J. Math. Oxford-Ser. (2) 36 (1985), pp. 183-213; Absolutely continuous spectra of second order differential operators with short range and long range potentials, SIAM J. Math. Anal 17 (1986), pp. 182-196.

[K] KLaus, Martin, Asymptotic behavior of Jost functions near resonance points for Wigner-von Neumann type potentials, J. Math. Phys. 32 (1991), pp. $163-174$

[L] Love, C. E., Singular integral equations of the Volterra type, Trans. Amer. Math. Soc. 15 (1914), pp. 467-476.

[LR] Landgren, J. J. and ReJto, P. A., An application of the maximum principle to the study of essential selfadjointness of Dirac operators. I, J. Math. Phys. 20 (1979), pp. 2204-2211.

[LRK] Landgren, J. J. and Rejto, P. A.; Appendix by Klaus, Martin, An application of the maximum principle to the study of essential selfadjointness of Dirac operators II, J. Math. Phys. 21 (1980), pp. 1210-1217.

[Ok] OkIKIOLu, G. O., "Aspects of the theory of bounded integral operators," Academic Press, 1975.

[O] Olver, F. J. W., "Asymptotics and Special Functions," Academic Press, 1974.

[P] Pearson, D. B., Scattering theory for a class of oscillating potentials, Helv. Phys. Acta 52 (1979), pp. 541-554. 
[R] REJTo, P. A., An application of the third order JWKB-approximation method to prove absolute continuity $I$. The construction, Helv. Phys. Acta 50 (1977), pp. 479-494; II. The estimates, Helv. Phys. Acta 50 (1977), pp. 495-508; On a theorem of Tichmarsh-Kodaira-Weidmann concerning absolutely continuous operators, I, J. Approx. Theory 21 (1977), pp. 333-351; On a theorem of Tichmarsh-Kodaira-Weidmann concerning absolutely continuous operators, II, Indiana Univ. Math. J. 25 (1976), pp. 629-658.

[RS] Reed, M. And Simon, B., "Analysis of operators, Methods of Modern Mathematical Physics, Vol. IV," Academic Press, 1978. See Section XIII.13, Example 1.

[RT] Rejto, P. and Taboada, M., Weighted resolvent estimates for Volterra operators on unbounded intervals, J. Math. Anal. Appl. 160, 1 (1991), pp. 223-235.

[Si] SibUYA, YasUTAKa, "Global Theory of a second order linear ordinary differential equation with a polynomial coefficient," North Holland Mathematical Studies, 18, 1975.

[St] Stolz, Günter, On the absolutely continuous spectrum of perturbed periodic Sturm-Liouville operators, J. Reine Angew Math. 416 (1991), pp. 1-23; Bounded solutions and absolute continuity of Sturm-Liouville operators, Johann Wolfgang Goethe-Universität Frankfurt, February 1991.

[VW] von Neumann, J. and Wigner, E., Über merkwürdige diskrete Eigenwerte, Phys. Z. 30 (1929), pp. 465-467.

[We] Weidmann, J., "Spectral Theory of Ordinary Differential Operators," SpringerVerlag Lecture Notes in Mathematics \# 1258, 1987.

[Wh] White, D. A. W., Schroedinger operators with rapidly oscillating central potentials, Trans. Amer. Math. Soc. 275 (1983), pp. 641-677. 